\title{
Hierarchies in concept attainmen in the selection mode with exemplar and non-exemplar start cards
}

\author{
LEONARD M. GIAMBRA, Miami University, Oxford, Ohio \\ 45056
}

The purpose of this study was to investigate the generality of the hierarchy of concepts to the selection mode with exemplar or nonexemplar start cards. The logical hierarchy of concepts in which lower level concepts form the components of higher level concepts was found to operate empirically in the context of the selection mode with a complete learning task. Furthermore, the Type of Start Card was found not to affect the relative difficulty of the levels of the hierarchy. Also noted was a faster time-per-trial for females over males and a Type Concept by Type Start Card interaction on per cent correct card choices.

Neisser \& Weene (1962) developed a hierarchy for concepts with one or two relevant dimensions. Level 1 concepts were the simplest and formed the components of Level 2 concepts which themselves formed the components of Level 3 concepts which are the most complex (see Table 1). Neisser and Weene demonstrated that this logical hierarchy was operating empirically. Their experiment was done within the reception mode (Giambra, in press) of concept identification, using the complete learning task. In the complete learning task $S$ determined both the relevant dimensions and the correct concept rule. However, no control was made of the nature of the start card (exemplar or nonexemplar) and it was not demonstrated that each $\mathrm{S}$ had an understanding of each of the 10 conceptual types.

Laughlin \& Jordan (1967), Giambra (in press), and Laughlin (1968) found that the hierarchy was not operating in the selection mode (Giambra, in press) with respect to the relative difficulty of the Both/neither and the Implication concepts. Thus, the generality of the concept hierarchy to the selection mode was opened to dispute. Since Laughlin and Jordan, and Giambra used the attribute identification task (in which $\mathrm{S}$ determines only the relevant dimension(s), knowing the correct rule) the source of the nongenerality of the hierarchy of concepts was confounded between task and experimental mode. The question arises as to whether Laughlin's as well as Giambra's results were due to a different task (attribute identification vs complete learning) or to a different mode of presentation (selection vs reception). For all Level 2 concepts the nature of the start card (exemplar or nonexemplar) determines the relative initial difficulty of the concepts. Specifically, conjunctive Level 2 concepts are more difficult with nonexemplar start cards than with exemplar start cards while the converse is true for disjunctive Level 2 concepts. Giambra (in press) however, found that in the selection mode with the attribute identification task Implication was more difficult than Both/neither with both types of start cards.

Considering the similarities and differences between Neisser \& Weene (1962), Laughlin \& Jordan (1967), Laughlin (1968), and Giambra (in press), it was evident that a study of the concept hierarchy in the selection mode with the complete learning task and controlling for type of start card was in order. Furthermore, to be sure that the results would be due entirely to the concepts themselves it was necessary to demonstrate that each $S$ understood all 10 concept types prior to entering the experiment. Such a study was done to determine the generalizability of the hierarchy of concepts to the selection mode and is reported here.

\section{SUBJECTS \\ The Ss were 20 volun teers from Miami University. PROCEDURE}

The experiment consisted of two phases. In the first phase Ss learned the 10 concept types. This phase consisted of 10 sessions of $1 / 2$ - to $1-h$ duration; one session was for each concept type. In each of these sessions each $S$ worked a total of eight problems in the selection mode. In each session the conceptual rule was carefully explained and examples of it were given. The explanation was followed by two problems in which $E$ answered S's questions while $S$ attempted to identify the relevant dimensions. In each of the remaining six problems, Ss were required to identify the concept under a criterion of four correct verbal guesses of the concept; an example of a verbal guess of a conjunction concept was "red one and green three." In the learning phase half the Ss had only exemplar start cards and half had only nonexemplar start cards. The second and experimental phase consisted of from one to four sessions of 1-h duration spaced no more than two days apart. In the first session it was explained to $S$ that unlike the practice phase any one of the 10 concept types might be the unknown concept of a particular problem. Each $S$ worked 20 problems. The 20 problems consisted of two of each of the 10 concept types, one which began with an exemplar and one with a nonexemplar. The order of the problems was determined by a Latin Square of size 20.

Each problem was solved within the framework of the following procedure: (a) The E first pointed out a stimulus card on the display board which was before $S$. This was the start card and $E$ verbally indicated its status as an exemplar or nonexemplar of the concept to be identified. (b) The $S$ then selected another stimulus instance which he felt would aid him in identifying the concept. (c) The $S$ indicated if he thought that that card was an exemplar of the concept. (d) The E revealed whe ther or not the card $S$ selected was an exemplar of the concept. (e) The $S$ took a verbal guess of the unknown concept. (An example might be "three circular figures.") Steps b through e were repeated until the problem was stopped by $E$. Each problem was stopped if the concept was guessed correctly on four consecutive trials or if 15 min passed, or after 20 trials, provided $S$ was not in the criterion sequence. The instructions merely indicated that $S$ was to identify the concept and that he could proceed at his own pace. All aspects of S's responses were recorded as well as time-per-problem. DESIGN

The design was a 2 by 2 by 10 factorial with repeated measures across the last two factors. The first factor was Sex and was included purely for control. The second factor was Type of Start Card; one problem with each concept type was begun with an exemplar and one was begun with a nonexemplar. The third factor was Type of Concept.

Table 1

Time Per Trial and Percent Correct Card Choices for Concepts of Hierarchy

Time Per Trial Percent Correct Card Choices Mean Exemplar Nonexemplar Start Card Start Card

\section{Level 1}

\begin{tabular}{|c|c|c|c|c|}
\hline Presence (A) & .35 & 82 & 69 & 76 \\
\hline Absence (-A) & .42 & 78 & 86 & 82 \\
\hline \multicolumn{5}{|l|}{ Level 2} \\
\hline Conjunction $(\mathrm{A} \cdot \mathrm{B})$ & .50 & 65 & 50 & 57 \\
\hline $\begin{array}{l}\text { Disjunctive Absence } \\
(-A v-B)\end{array}$ & .60 & 70 & 76 & 73 \\
\hline Disjunction (AvB) & .51 & 70 & 72 & 71 \\
\hline $\begin{array}{l}\text { Conjunctive Absence } \\
(-A \cdot-B)\end{array}$ & .52 & 71 & 68 & 70 \\
\hline Implication (-AvB) & .59 & 65 & 71 & 68 \\
\hline Exclusion $(-A \cdot B)$ & .62 & 74 & 62 & 68 \\
\hline \multicolumn{5}{|l|}{ Level 3} \\
\hline $\begin{array}{l}\text { Both/neither } \\
(A \cdot B) v(-A \cdot-B)\end{array}$ & .69 & 65 & 57 & 61 \\
\hline $\begin{array}{l}\text { Either/or } \\
(-A \cdot B) v(A \cdot-B)\end{array}$ & .73 & 64 & 58 & 61 \\
\hline
\end{tabular}




\section{STIMULUS MATERIAL}

The stimulus material used in the first phase consisted of eight stimulus cards mounted on a display board. The stimulus cards varied along three binary dimensions and thus represented all possible combinations of those dimensions. Three sets of eight stimulus cards were used. The stimulus material in the experimental phase consisted of 27 stimulus cards mounted on a single display board. The 27 stimulus cards varied along three ternary valued dimensions and thus represented all possible combinations of the three dimensions. Stimulus cards were randomly arranged on the display boards. The nature of all stimulus material was explained to all Ss. RESULTS AND DISCUSSION

Two dependent measures are reported: time-per-trial and per cent correct card choices. The time-per-trial was the total time-per-problem divided by the trials for that problem. Timeper-trial was used instead of trials-per-problem or time-perproblem because problems were stopped after 20 trials or after $15 \mathrm{~min}$, thus distorting these two dependent measures. Per cent correct card choices was simply the per cent of trials in which $S$ correctly guessed the status (exemplar or nonexemplar) of the stimulus card selected. Analyses of variance were performed on each of these measures. With the time-per-trial measure, Type Concept $[F(9,108)=4.42, p<.01]^{2}$ and $\operatorname{Sex}[F(1,12)=6.67$, $\mathrm{p}<.05]$ produced significant effects. For per cent correct card choices Type Concept $[F(9,108)=5.86, p<.01]$ and the Type Concept by Type Start Card interaction $[F(9,108)=2.42$, $\mathrm{p}<.05]$ produced significant effects. The Sex main effect was the result of a faster time-per-trial $(.47 \mathrm{~min}$ ) for females than for males (.61 min). Table 1 portrays the Type Concept main effect and the Type Concept by Type Start Card interaction. The relative difficulty of the three concept levels is quite apparent for both measures except for the anomaly of the conjunctional concept with the per cent correct card choices measure. Why Ss should do so poorly with respect to per cent correct card choices for the conjunctional concept was unexplainable from a reexamination of the S protocols. The Type Concept by Type Start Card interaction resulted from a reduction in per cent correct card choices with the nonexemplar start card for Level 3 concepts and conjunctive type Level 2 concepts as well as the Presence concept of Level 1 and an increase in per cent correct card choices for the disjunctive type Level 2 concepts and the Absence concept of Level 1.

Using the time-per-trial measure every type of concept was directly compared with every other type. The comparisons, made separately for problems begun with the two types of start cards, were in terms of the proportion of Ss who found one type easier than the other. Most proportions were based on slightly fewer than 20 Ss since those who found the two problems equally difficult were not counted. Also reducing the base for some concept types was the fact that no data was available for a particular $S$ on a particular problem because the problem was discarded due to an E's error. ${ }^{3}$ For each comparison, the null hypothesis is that the two concepts were equally difficult, and that the proportion differs from one-half only by chance. In all those cases where the comparison is between concepts of different levels, we have the alternate hypothesis that an $S$ is more likely to find the lower level concept easier. For the exemplar start card, 23 of 28 interlevel comparisons were in the predicted direction while with the nonexemplar start card all 28 comparisons were in the predicted direction. Of the 51 comparisons in the predicted direction, 25 were significantly $(p \leqslant .05)$ different from one-half. Thus, it appears that the level of complexity also plays an important role in determining the difficulty of concept type in the context of the selection mode regardless of the nature of the start card. Therefore, one sees that the differential memory requirement of the selection mode with a complete learning task, as well as the requirement that $S$ actively seek out information, has no moderating influence with respect to the hierarchy of concepts. Furthermore, contrary to the finding of Laughlin \& Jordan (1967), Laughlin (1968), and Giambra (in press), the relative difficulty of Implication and Both/neither concepts remains as predicted by the hierarchy. This experiment used the complete learning task as did Neisser \& Weene (1962), while Laughlin \& Jordan (1967), Laughlin (1968), and Giambra (in press) used an attribute identification task; thus it seems that the added task of determining the correct concept rule increased the difficulty of Both/neither concept much more than it did for the Implication concept. Further support for this contention is found by referring to the timesper-trial for the 10 concept types in the training sessions (Phase 1) which used the attribute identification task. The time-per-trials in minutes for the 10 concepts were as follows from lowest to highest: Presence (.19), Disjunctive Absence (.22), Absence (.23), Conjunction (.24), Exclusion (.29), Both/ neither (.31), Conjunctive Absence (.33), Either/or (.38), Disjunctive Absence (.39), Implication (.44). No Type Concept by Type Start Card interaction was found with the time-per-trial measure. However, that interaction was found operating using a trials-per-problem measure. The trials-per-problem measure revealed Implication more difficult than Both/neither with exemplar start cards (which was what Laughlin used) but the converse was true with nonexemplar start cards. Giambra (in press) found Implication more difficult than Both/neither regardless of start card. The source of this difference between the present study and Giambra (in press), Laughlin (1968), and Laughlin \& Jordan (1967) could be that in this study Ss worked (in Phase 1) 10 times as many problems of all concept types and thus acquired greater facility in solving concept problems making them more able to solve the concept problems nearer the minimum number of trials permitted by the start card.

Intralevel comparisons of concepts revealed for the most part proportions near one-half indicating equal concept difficulty; this replicates Neisser \& Weene (1962). There was one notable exception, however; Presence (A) was much easier than Absence (-A) with the exemplar but not with the nonexemplar start card. This was directly opposite Neisser and Weene's finding of Absence easier than Presence. Also in this study and nonsupportive of Neisser and Weene was the finding that the Implication and Disjunctive Absence concepts were not in general significantly more difficult than other Level 2 concepts.

\section{REFERENCES}

GIAMBRA, L. M. Effect of number irrelevant dimensions with 10 concept types on the attribute identification task in the selection mode with exemplar and nonexemplar start cards. Psychonomic Science, in press.

LAUGHLIN, P. R. Focusing strategy for eight concept rules. Journal of Experimental Psychology, 1968, 77, 661-669.

LAUGHLIN, P. R., \& JORDAN, R. M. Selection strategies in conjunctive, disjunctive, and biconditional concept attainment. Journal of Experimental Psychology, 1967, 188-193.

NEISSER, U., \& WEENE, P. Hierarchies in concept attainment. Jou mal of Experimental Psychology, 1962, 282-290. NOTES

1. This report is based on part of a dissertation submitted to The Ohio State University.

2. Note that the df is 108 instead of 162 because three Ss from each sex were not included in the ANOVA due to an E error on one or two problems in the experimental sessions.

3. The table of proportions may be obtained from the author upon request. 